\title{
Propellant Ionization and Mass Spectral Measurements in the Plume of an SPT-100
}

\author{
Lyon B. King* and Alec D. Gallimore ${ }^{\#}$ \\ Plasmadynamics and Electric Propulsion Laboratory \\ Department of Aerospace Engineering \\ University of Michigan \\ Ann Arbor, MI 48105
}

\begin{abstract}
Knowledge of the ion species emitted by the SPT-100 Hall thruster provides considerable insight to the basic thruster performance. In order to gain detailed information concerning the composition of the plume plasma a custom molecular beam mass spectrometer was constructed to interrogate the exhaust plume. An investigation using this technique yielded both the ionization fraction of the xenon propellant as well as a qualitative analysis of the minority plasma species attributed to ground-test facility interactions. The plasma was found to consist of $89 \% \mathrm{Xe}^{+}, 11 \% \mathrm{Xe}^{2+}$, and $0.2 \% \mathrm{Xe}^{3+}$. The existence of parasitic facility gasses including nitrogen, oxygen and water vapor was documented, as well as trace carbon ions caused by sputtering of graphite surfaces in proximity of the thruster. Through a species-dependent analysis of the ion energy distribution the products of momentum transfer collisions between plume ions were uncovered. These products appeared as highenergy tails on the ion voltage distribution function representing ions at voltages greater than that applied to the thruster discharge.
\end{abstract}

\section{Nomenclature}

$\begin{array}{ll}\mathrm{I} & \text { Ion current (A) } \\ \mathrm{V} & \text { Voltage }(\mathrm{V}) \\ \mathrm{q}_{\mathrm{i}} & \text { Integer ion charge state } \\ \mathrm{m}_{\mathrm{i}} & \text { Ion mass }(\mathrm{kg}) \\ \mathrm{E} & \text { Ion energy (J or eV) } \\ \mathrm{f}(\mathrm{E} / \mathrm{q}) & \text { Ion voltage distribution }(\mathrm{s} / \mathrm{m}) \\ \mathrm{d}_{\text {gate }} & \text { Gate electrode length }(\mathrm{m}) \\ \mathrm{t}_{\mathrm{gate}} & \text { Gate opening duration }(\mathrm{s}) \\ \mathrm{t}_{\text {taf }} & \text { Ion time-of-flight }(\mathrm{s}) \\ \mathrm{M}_{\mathrm{i}} & \text { Ion atomic mass number (amu) } \\ \mathrm{m}_{\mathrm{p}} & \text { Proton mass }(\mathrm{kg}) \\ \mathrm{e} & \text { Elementary charge }(\mathrm{C}) \\ \mathrm{D}_{\mathrm{s}} & \text { MBMS inlet skimmer dia. (mm) } \\ \mathrm{D}_{\text {coll }} & \text { MBMS collimator dia. }(\mathrm{mm})\end{array}$
$\mathrm{r} \quad$ Radial distance from thruster $(\mathrm{m})$
$\theta \quad$ Angle (deg)
$\mathrm{t} \quad$ Time $(\mathrm{s})$
$\mathrm{G}_{\mathrm{CEM}}$ Electron multiplier gain
$\mathrm{n}_{\mathrm{i}}$ Ion density $\left(\mathrm{m}^{-3}\right)$
$u_{i}$ Ion velocity $(\mathrm{m} / \mathrm{s})$
$\alpha_{n} \quad$ Voltage-dependent frac. of $\mathrm{Xe}^{\mathrm{n}+}$
$\Phi_{\mathrm{n}} \quad$ Global flow fraction of $\mathrm{Xe}^{\mathrm{n}+}$
$\mathrm{E}_{\text {coll }} \quad$ Energy of collision ( $\mathrm{J}$ or $\mathrm{eV}$ )
$\mathrm{m}_{1,2}$ Mass of species $1,2(\mathrm{~kg})$
$\mathrm{E}^{\prime}$ Post-collision energy $(\mathrm{J}$ or $\mathrm{eV})$

Copyright $\odot 1998$ by Lyon B. King. Printed by the American Institute of Aeronautics and Astronautics with Permission.

* Research Associate, Member AIAA. Current address: NIST, Time \& Frequency Div. 847 325 Broadway, Boulder, $\mathrm{CO} 80303$

\# Associate Prof., Senior member AlAA. 


\section{Introduction}

In-situ plasma probes constitute a simple method through which detailed plasma properties can be evaluated. However, these probes provide no insight to plasma species (charge state and mass) composition. Indeed, the RPA technique of measuring the ion energy distribution function is not merely insensitive to flow species, rather its interpretation is only valid for a single species. Although RPAs enjoy widespread use in plasma diagnostics it must be recognized that in a multiple-species flow differentiation of the $\mathrm{I}(\mathrm{V})$ vs $\mathrm{V}$ data does not produce a function directly proportional to the energy distribution as widely accepted, instead

Eqn. 1

$$
\frac{d I}{d V} \propto \frac{q_{i}^{2}}{m_{i}} f\left(E / q_{i}\right) .
$$

The non-trivial relationship between the RPA data and the ion energy places a limitation on the rigor with which the resultant quantities can be applied. ${ }^{1}$

Further complicating the understanding of RPA data has been the extensive documentation of a high-energy "tail" of ions with accelerating voltages much greater than that applied between the anode and cathode of the Hall thruster. This tail has been the subject of much controversy and confusion of late; postulates to explain its existence included plasma instability-driven turbulence within the thruster discharge as well as recombinative or charge-changing collisions within the exhaust plume. Exploration of these hypotheses required species-dependent analysis of the plasma. In addition to the data interpretation issues, the numerical differentiation required with the RPA technique produced unavoidably "noisy" distribution functions that impose further uncertainties in data analysis.

As a means of quantifying the plasma species constitution and to obtain a verification of the puzzling ion energy distribution, Manzella utilized spectroscopic techniques to interrogate the Hall thruster plasma. In these studies emission spectroscopy was used to measure the xenon ionization fractions ${ }^{2}$ while a laser-induced fluorescence (LIF) technique was used to obtain the $\mathrm{Xe}^{+}$velocity distribution function. ${ }^{3}$ The emission study suggested that the fraction of $\mathrm{Xe}^{+}$was between $76 \%$ and $89 \%$ of the total flow while $\mathrm{Xe}^{2+}$ comprised between $19 \%$ and $12 \%$. However, the author acknowledged that the Boltzmann equilibrium model used to derive these values from the data was possibly not well-suited to the Hall thruster plasma. Although the correct model was identified as a collisional-radiative equilibrium (CRE) model, no such model was developed for several reasons attributed to the complicated atomic energy structure of xenon coupled with a lack of experimental data regarding various excitation rates. The LIF investigation produced accurate values of the average ion velocity. Additionally, the shape of the fluorescence excitation spectrum was evaluated to assess other plasma parameters. However, the results implied by this shape analysis were in striking disagreement with the RPA data: the LIF study implied a width in the distribution function corresponding to a $3 \mathrm{eV}$ spread in ion energy while the RPA data implied a spread on the order of $100-150 \mathrm{eV}$.

The state of research preceding the study reported here reflected conflicting and uncertain information regarding the ionic composition and energy within the Hall thruster plume: the only species analysis performed required the use of a complex model to achieve estimates of propellant ionization fraction, while the two existing measurements of ion 
energy distribution were in disagreement. It was apparent that a technique was required to (1) provide a direct measure of the propellant ionization fraction independent of a model regarding the plasma equilibrium state and (2) directly measure the energy distribution function of each species using a method that is sensitive to ionic charge and mass. Based on this need the construction of a custom-built molecular beam mass spectrometer for Hall thruster plume studies was initiated.

\section{Description of Apparatus}

The MBMS system used a set of orifice skimmers to admit a beam of plume ions from the main vacuum chamber into an array of differentially pumped sub-chambers. The instrument is shown in Figure 1 and Figure 2. The sub-chambers were maintained at high vacuum to minimize and effectively eliminate collisions involving ions within the beam. A sampling skimmer orifice was mounted on the upstream end of the MBMS; this orifice skimmed off a small diameter ion beam into the first sub-chamber. This beam was then collimated by a second orifice at the downstream end of the first sub-chamber. The collimated beam then passed through the entrance slit of a 45-degree electrostatic energy analyzer. This analyzer employed a constant electric field such that only ions with a preselected voltage (energy per charge, since $V=E / q$ ) have a trajectory which permits them to traverse the exit slit and impinge upon a ceramic channel electron multiplier (CEM). By recording the output current of the CEM as a function of the electric field strength within the 45-degree analyzer the ion energy distribution function was evaluated. A more detailed discussion of the energy analyzer can be found in Reference 1 and 4.

Mass analysis was obtained by using an electrostatic beam gate to "chop" the ion beam immediately downstream of the inlet skimmer and record the time required for an ion to pass from the gate to the detector: since the 45-degree analyzer admits only ions with a pre-selected value of $m_{i} u_{i}{ }^{2} / 2 q$ to the CEM, ions of different mass but the same energy-percharge will have different velocities and, hence, will arrive at the detector at different times. The configuration of the instrument was based on that of Pollard. ${ }^{5}$ The beam gate consists of two planar electrodes of length $\mathrm{d}_{\text {gate }}$ placed on opposing sides of the ion beam. A large voltage difference (around $1.5 \mathrm{kV}$ ) is applied between the plates creating a strong electric field perpendicular to the ion beam line. This field deflects all ions such that their trajectories intersect the chamber wall. At time $t=0$ this electric field is removed for a period of time lasting $\mathrm{t}_{\text {gate }}$, opening the gate and admitting a pulse of ions to the field-free drift region of length $\mathrm{d}_{\text {tof }}$. According to the ionic mass-to-charge ratio, each ion species arrives at the CEM a time $t_{\text {tof }}$ after the start of the gate pulse according to

Eqn. 2

$$
\frac{M_{i}}{q_{i}}=\frac{d_{\text {tof }}^{2}}{t_{\text {tof }}^{2}} \frac{2 \mathrm{eV}_{\mathrm{i}}}{m_{\mathrm{p}}}
$$

In Eqn. 2 the drift length, $\mathrm{d}_{\text {tof }}$, can be easily measured, the ion voltage, $\mathrm{V}_{\mathrm{i}}$, is determined by the 45 -degree analyzer, and $\mathrm{m}_{\mathrm{p}}$ is the mass of a proton (constant). Thus, by measuring the flight time, $t_{\text {tof }}$, the ion mass-per-charge is easily obtained. A high-speed transimpedance preamplifer (Amptek A250) was used to convert the CEM output current to voltage; this voltage was then post-amplified using a fast op-amp circuit (Analog Devices AD829). Combining the gain of the CEM with the amplifier circuitry yields an overall system gain of $2.5 \times 10^{14}$ V/A for the ion beam current. A much more detailed description 
of this apparatus and the defining design constraints can be found in Reference $1 . \quad$ A summary of the MBMS physical parameters is presented here as Table 1.

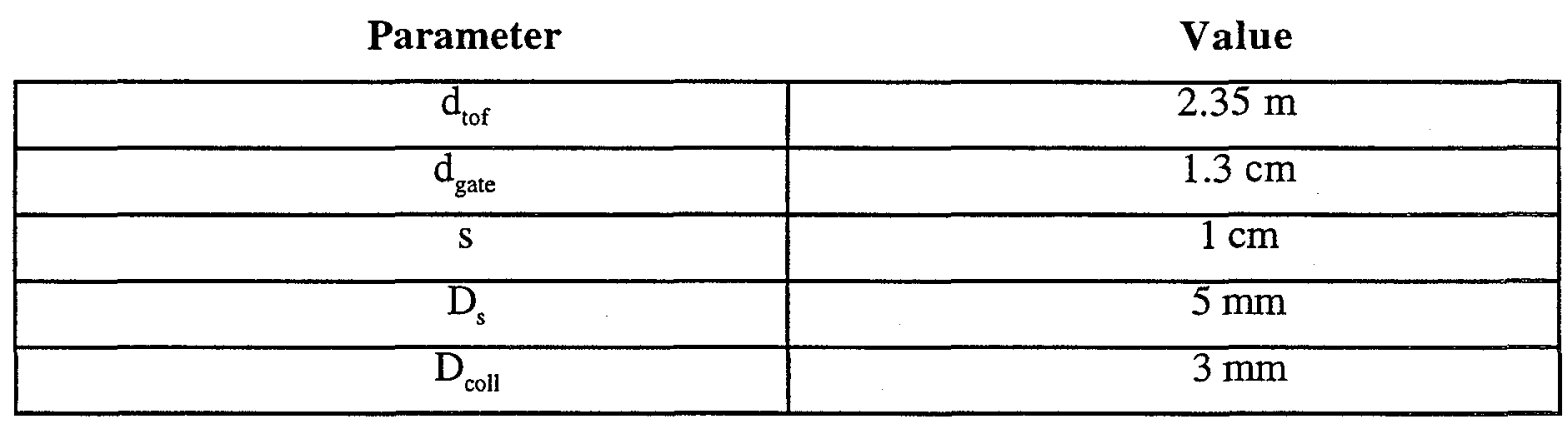

Table 1. Physical parameters of MBMS time-of-flight components.

Both the sampling and collimating skimmers were fashioned from 304 SS plates drilled and countersunk from the downstream face. This created a very thin-edged orifice to minimize skimmer wall effects. The sub-chambers were constructed from standard conflat vacuum hardware composed of eight-inch-inner-diameter 304 SS tubing, with the exception of the portion of the beam forming chamber lying within the main vacuum tank which was constructed of six-inch-I.D. tubing. The first sub-chamber was evacuated by a ten-inch-diameter oil diffusion pump (Varian model HS-10) operating on Dow-Corning 705 vacuum fluid and the chamber housing the electrostatic energy analyzer was evacuated using a six-inch-diameter oil diffusion pump (Varian model M6) also operating on DowCorning 705. Both oil diffusion pumps were fitted with conductively cooled "halo" baffles at the inlet to reduce oil backstreaming into the MBMS volume. The two diffusion pumps were backed by an 80 -cfm rotary mechanical pump (Kinney model $\mathrm{KDH}-80$ ). The MBMS vacuum was monitored by one thermocouple pressure sensor and two hot-cathode ionization pressure gauges; this system enabled an internal base pressure within the MBMS system of $3 \times 10^{-7}$ torr which was achievable after approximately one hour of pumping. A large diameter (eight-inch) stainless-steel gate valve was placed between the energy analyzer chamber and the main vacuum tank enabling rapid venting and re-pumping of the MBMS for configuration adjustments without compromising the main tank vacuum.

\section{Experimental Set-up}

The SPT-100 was mounted to a rotary table such that the rotation axis coincided with the center of the exit plane of the thruster. Therefore, by rotating the thruster relative to the fixed MBMS skimmer inlet the plasma plume could be sampled as a function of angular position at a fixed radial distance, $r$, from the exit plane. This set-up is illustrated schematically in Figure 3 with a photograph of the setup shown in Figure 4. The circuitry used to obtain the time-of-flight spectra and convert the data into mass-per-charge spectra is shown in Figure 5.

The centerline (thrust axis) of the thruster was denoted as zero degrees, with positive theta values representing points in the cathode half-plane of rotation (the angular position shown in Figure 3 represents $\theta=+90$ degrees). The angular alignment of the thruster and MBMS was achieved by using a laboratory laser to establish the MBMS beam line. The laser beam line was used to verify the angular orientation of the 45-degree 
electrostatic analyzer to within 0.5 degrees; similarly, the thruster was rotated such that the laser beam line was precisely aligned with the center of the exit plane of the thruster, as shown in Figure 3, establishing the 90 degree position of the SPT-100 to better than 0.5 degrees. Since the relative uncertainty in angular position of the rotary table was 0.1 degrees, the uncertainty in position for all data points is,+-0.5 degrees due to initial alignment uncertainty.

The inlet of the MBMS was grounded, while the plasma maintained a potential which was somewhat higher than ground. The artificial energy increase imposed on the ions as they fell from plasma potential to ground potential was subtracted off of the data through post-processing. The magnitude of the correction was obtained through the use of a Langmuir probe immediately upstream of the MBMS inlet.

\section{Results}

As a demonstration of the data interpretation principles for the TOF system Figure 6 shows a typical TOF spectra obtained for a 45-degree analyzer ion pass voltage of $280 \mathrm{~V}$ at a position of $0.5 \mathrm{~m}$ radius and 5 degrees off axis. The potential difference across the gate electrodes was maintained at $1.5 \mathrm{kV}$ nominally. At time $t=0$ this potential difference was removed with a rise time of $40 \mathrm{nsec}$, opening the ion gate for a time $t_{\text {gate }}=5 \mu \mathrm{sec}$. This high-voltage transient induced "shot noise" into the facility ground plane which appeared as a noise burst in the CEM amplifier circuitry; this noise rapidly decayed after the pulse returning to a zero-current baseline. The transient current peaks appearing in the CEM output signal represent the arrival of different ion species at the detector. For example, from Eqn. 2 for $280 \mathrm{~V}$ ions, singly ionized xenon with $\mathrm{M}_{\mathrm{i}} / \mathrm{q}_{\mathrm{i}}=131 \mathrm{amu}$ would take 120

$\mu$ sec to travel the $2.35 \mathrm{~m}$ path and arrive at the detector; this species is seen as the dominant peak. Doubly ionized xenon has $\mathrm{M}_{\mathrm{i}} / \mathrm{q}_{\mathrm{i}}=65.5$ and therefore corresponds to the peak seen at $t=85 \mu \mathrm{sec}$. The conversion between time and mass-per-charge can be performed yielding a mass spectra as shown in Figure 7. In this figure the baseline offset displayed after an intense current peak was due to pre-amplifier undershoot.

This mass spectra shows the first three ionization states of the propellant, xenon, along with a small population of light gasses with amu less than 30. Many of the characteristics illustrated in this spectra are typical of all data points. As a result of the quadratic conversion between arrival time and species mass the heavier mass peaks, although equal width in time, are wider in terms of amu than the lighter masses. Although the peak widths are on the order of $10 \mathrm{amu}$, this width does not impose any difficulty with mass identification or propellant ions: the leading (left) edge of the mass peak marks the arrival time of the species and, therefore, the species mass.

In an attempt to better identify the light gasses evidenced in Figure 7 the gate pulse was narrowed and the oscilloscope was configured to obtain better resolution for these minor species. However, the extremely short gate pulse duration necessary to resolve the light gasses negates any quantitative information that can be obtained from the species peak height. This principle is fully explained in Reference 1. Regardless of the ambiguous peak height, though, the species M/q can still be accurately identified to provide an analysis of the plasma components. The resulting minor species spectra is shown in Figure 8.

Based on the problems involved with deriving quantitative information for light gas species it was decided to limit all quantitative analysis to the ionization states of the heavy propellant, xenon. Thus, mass spectra were obtained at the fixed position of $0.5 \mathrm{~m}, 5$ 
degrees, as a function of ion voltage with the gate pulse duration sufficient to resolve the ionization states of xenon with full transmission of current peaks. This was accomplished by using the 45-degree analyzer to define the pass voltage and taking a TOF mass spectra for only ions with this voltage. In order to avoid possible confusion it is emphasized that the thruster operation parameters of $300 \mathrm{~V}$ applied discharge at $4.5 \mathrm{~A}$ were not changed during testing; the voltages indicated for the TOF spectra correspond to the ion energy/q being analyzed in the MBMS for the fixed thruster discharge. Figure 9 illustrates the mass spectra obtained for ions with voltages of 168, 268, and $368 \mathrm{~V}$ at the fixed SPT-100 operating point of $300 \mathrm{~V}$ at $4.5 \mathrm{~A}$. In this plot it is clear that the low-energy ion population is dominated by $\mathrm{Xe}^{2+}$ (over $60 \%$ of the ions at $168 \mathrm{~V}$ are doubly charged), whereas the high-energy ions are mostly $\mathrm{Xe}^{+}$. It should be noted that in Figure 9 no attempt was made to obtain narrow peaks of high mass resolution; on the contrary, the gate pulse duration was set excessively long in order to ensure the full transmission of the ion current pulses such that the peak height indicated a true measure of ion density fraction.

\section{Discussion}

\subsection{Minor Species Analysis}

The spectra obtained for identification of the minor species, shown in Figure 8, shows results consistent with previous investigations. In an emission spectroscopic study Manzella documented clear evidence of the ingestion and ionization of background gas from the vacuum facility within the SPT-100 discharge. ${ }^{2}$ Due to facility pumping imperfections a trace amount of parasitic background gas exists within the chamber during testing. This gas consists mainly of atmospheric components, i.e. nitrogen and oxygen, with a disproportionate amount of water vapor. The neutral background gas diffuses into the thruster discharge chamber where it is ionized and accelerated back out with the propellant ions. Manzella estimated a quantity of entrained background ions equivalent to $2 \%$ of the main propellant flow.

The minority species evaluation reported here supports the finding of ingested facility gasses. Due to the molecular "cracking" signature of the water molecule, electron impact ionization of this species produces dissociated peaks at $\mathrm{M} / \mathrm{q}=18\left(\mathrm{H}_{2} \mathrm{O}^{+}\right), 17\left(\mathrm{OH}^{+}\right)$, $2\left(\mathrm{H}_{2}^{+}\right)$, and $1\left(\mathrm{H}^{+}\right)$. Likewise, an electron impact ionization reaction involving molecular nitrogen and oxygen would most likely produce atomic ions: the reason for this is that the bond energy of the diatom $\left(5 \mathrm{eV}\right.$ for $\mathrm{O}_{2}$ and $9 \mathrm{eV}$ for $\mathrm{N}_{2}$ ) is much less than the ionization potential of the molecule $\left(12 \mathrm{eV}\right.$ for $\mathrm{O}_{2}$ and $15.6 \mathrm{eV}$ for $\left.\mathrm{N}_{2}\right){ }^{6}$ Thus electron impacts are much more likely to dissociate the diatom into constituent neutral atoms (which are subsequently ionized) than direct ionization of the molecule. The existence of singly ionized carbon in the plume signature arises from facility effects, as well. In order to prevent material damage to the vacuum chamber walls immediately behind the MBMS inlet skimmer due to impacting high-energy ions these surfaces were extensively coated with low-sputter-yield flexible graphite sheets. Evidence of substantial ablative sputtering of the graphite was apparent as a thin gray film deposited on metallic facility surfaces during posttest inspections and re-configurations; this graphite is the likely source of the $\mathrm{M} / \mathrm{q}=12$ signature in the minority spectrum.

It is important to note that the spectroscopic study by Manzella found no evidence of singly ionized nitrogen in the emission spectra, although clear evidence of $\mathrm{N}_{2}^{+}$was documented. This is contrary to the mass spectra obtained in this study showing a substantial peak at $\mathrm{M} / \mathrm{q}=14$ with a negligible signature at $\mathrm{M} / \mathrm{q}=28$. There are two 
possible explanations for this disagreement: (1) the facility background pressure in the Manzella study was an order of magnitude lower than the pressure in the study reported here possibly causing trace amounts of $\mathrm{N}^{+}$to be below the optical detection limit; and (2) the gate pulse duration utilized to obtain the minority spectra reported here may have too short to permit the heavy $\mathrm{M} / \mathrm{q}=28$ ion to successfully be detected, while allowing the lighter $\mathrm{N}^{+}$ion to pass through the MBMS.

\subsection{Propellant Ionization}

Analysis of the propellant ionization state was accomplished by recording individual mass spectra for ion voltages ranging from $100 \mathrm{~V}$ up to $620 \mathrm{~V}$ at $20 \mathrm{~V}$ increments. Such a data set enabled the ionization fraction of each xenon species to be calculated as a function of ion voltage. The ion species current peaks are related to their respective ion densities through the value of ion charge state: the current output of the CEM for a given ion voltage and charge state is

Eqn. 3

$$
I_{i}\left(V_{i}, q_{i}\right)=G_{C E M} e_{i} u_{i}\left(V_{i}, q_{i}\right)
$$

noting that although $u_{i}$ is a function of $q_{i}$, the CEM acts as an ion counter rather than a charge counter so that $I_{i}$ is not directly proportional to $q_{i}$. Expressing the ion velocity in terms of ion charge state and voltage yields

Eqn. 4

$$
I_{i}\left(V_{i}, q_{i}\right)=G_{C E M} e_{i} \sqrt{\frac{2 q_{i} e V_{i}}{m_{i}}}
$$

Thus for a given species current peak the density and the current are related according to

Eqn. 5

$$
\mathrm{n}_{\mathrm{i}}\left(\mathrm{q}_{\mathrm{i}}\right) \propto \frac{\mathrm{I}_{\mathrm{i}}\left(\mathrm{q}_{\mathrm{i}}\right)}{\sqrt{\mathrm{q}_{\mathrm{i}}}} .
$$

Defining the number density fraction of $X e^{n+}$ having energy/q $=V_{i}$ as $\alpha_{n}\left(V_{i}\right)$ yields

Eqn. 6

$$
\alpha_{n}\left(V_{i}\right)=\frac{I\left(V_{i}, n\right) / \sqrt{n}}{\sum_{q}^{I}\left(V_{i}, q\right) / \sqrt{q}}
$$

In the analysis of the propellant mass spectra xenon ions up to $q=3$ were readily measured, with no conclusive evidence of $\mathrm{Xe}^{4+}$ exhibited in the spectra; complicating the search for $\mathrm{Xe}^{4+}$ was the fact that the mass-per-charge of this ion is $32.2 \mathrm{amu}$, which is very close to the $32 \mathrm{amu}$ attributable to singly ionized $\mathrm{O}_{2}$ present due to parasitic facility gasses. Therefore the mass spectra were inconclusive in identifying quadruply ionized xenon. The values of $\alpha_{n}$ were calculated from an assembly of mass spectra obtained at $20 \mathrm{~V}$ intervals of ion voltage recorded over a range from $100 \mathrm{~V}$ to $620 \mathrm{~V}$ (such as those shown in Figure 9 for ion voltages of 168,268 , and $368 \mathrm{~V}$ ). From each mass spectra "snapshot" at a given ion voltage, $\alpha_{n}$ was computed according to Eqn. 6 (for example the $168 \mathrm{~V}$ ion mass spectra shows $\mathrm{Xe}^{2+}$ to comprise approximately $60 \%$ of the flow); the results are compiled as Figure 10 , which shows a plot of the number density fraction of the first three ionization states of 
xenon plotted as a function of ion voltage. Compared with this plot is the total (species independent) ion voltage distribution function as measured in Reference 4.

Figure 10 indicates that the majority of the plume ions have undergone accelerations through approximately $270 \mathrm{~V}$; of these ions almost $90 \%$ are singly ionized. Although the ion density decreases for voltages less than $270 \mathrm{~V}$, a greater fraction of these low-voltage ions are multiply charged with nearly $100 \%$ of the $110 \mathrm{~V}$ ions consisting of $\mathrm{Xe}^{2+}$. The number fraction of $\mathrm{Xe}^{3+}$ peaks at about $6 \%$ of the ions having voltages of $150 \mathrm{~V}$. For voltages greater than the most probable voltage the fraction of multiply charged ions experiences a slight increase over the composition at $270 \mathrm{~V}$.

The total number density fractions for all ions in the plume can be obtained by integrating the voltage-dependent flow fractions over all voltages. Denoting the overall fraction of ions with charge $q_{i}=n^{+}$as $\Phi_{n}$,

Eqn. 7

$$
\Phi_{\mathrm{n}}=\frac{\int_{0}^{\infty} \alpha_{\mathrm{n}}(\mathrm{V}) \mathrm{I}(\mathrm{V}) \mathrm{dV}}{\int_{0}^{\infty} \mathrm{I}(\mathrm{V}) \mathrm{dV}}
$$

The total flow fractions computed from the MBMS data according to Eqn. 7 and the data of Figure 10 are compared with the fractions calculated by Manzella using an optical emission spectroscopic technique at the thruster exit plane; this comparison is shown in Table 2.

$$
\text { Species } \quad \Phi_{n} \text { from MBMS } \quad \Phi_{n} \text { from Manzella }{ }^{2}
$$

\begin{tabular}{|c|c|c|}
\hline $\mathrm{Xe}^{+}$ & 0.888 & 0.89 \\
\hline $\mathrm{Xe}^{2+}$ & 0.110 & 0.119 \\
\hline $\mathrm{Xe}^{3+}$ & 0.002 & (not measured) \\
\hline
\end{tabular}

Table 2. Comparison between MBMS-measured ionization fractions with values derived from Manzella's study using optical emission spectroscopy. Uncertainty in MBMS values is approximately $5 \%$.

The agreement between the two techniques demonstrated in the table is excellent, exhibiting only negligible differences. Additionally, the MBMS system provided the first ever documentation of the existence of $\mathrm{Xe}^{3+}$ within the Hall thruster plume.

Further insight into the propellant ionization and acceleration mechanism is possible through analysis of the TOF spectra. As discussed earlier and evidenced by Eqn. 1 the existence of multiple ion species presents considerable difficulty in determining the ion energy distribution function using a method insensitive to ion charge state. However, by compiling the species current peak heights in the TOF spectra as a function of ion voltage it is possible to construct true ion energy distribution functions independently for each ion species in the flow. These data are shown in Figure 11.

The species-dependent energy distributions display some subtle, yet remarkable features. The highest most-probable voltage is displayed by $\mathrm{Xe}^{+}$, with $\mathrm{V}_{\mathrm{m}}=274 \mathrm{~V}$, with the peaks in the multiple ions at lower voltages. This finding is consistent with the 
ionization and acceleration processes believed to exist within the thruster discharge chamber. It has been widely documented that the electron temperature within the discharge chamber attains a maximum in the region of highest magnetic field strength, which occurs very near the thruster exit plane. ${ }^{7,8}$ Indeed, this behavior was displayed in the model by Baranov; Figure 12 shows the results of this model in predicting neutral density,electron temperature, and plasma electric potential.

The energy required for the first ionization of neutral xenon is $12.1 \mathrm{eV}$, while the second ionization potential is $21.21 \mathrm{eV}$. ${ }^{6}$ Therefore, as the neutral atoms travel from the anode towards the exit plane the initial electron collisions will have sufficient energy for single ionization, but insufficient energy to form a multiple ion; formation of the multiply charged ions will occur further downstream where the electrons are hotter and, as a result of the distribution of plasma potential, experience less accelerating voltage. The ionization potential of $\mathrm{Xe}^{3+}$ is $32.1 \mathrm{eV}$. This would imply that the most-probable voltage of the triple ion should be less than that of the double. However, Figure 11 shows the distribution peak for the triple ion to be $255 \mathrm{~V}$ while the double exhibits a peak at $235 \mathrm{~V}$. This behavior is contrary to the ionization and acceleration mechanism described above. The reason for this anomaly is believed to stem from uncertainty in determining the peak heights corresponding to $\mathrm{Xe}^{3+}$ in the TOF spectra compiled as Figure 11: since the triply charged xenon ion represented only $0.2 \%$ of the total mass flow the current peaks were extremely small and, occasionally difficult to resolve in the TOF spectra. Combined with the $20 \mathrm{~V}$ resolution obtained by acquiring TOF spectra in $20 \mathrm{~V}$ intervals, this uncertainty could be responsible for a $20 \mathrm{~V}$ uncertainty in most-probable voltage location for the $f(V)$ curve.

\subsection{Ion Collision Evidence}

The ion energy distribution functions reported in this study along with previous measurements of ion energy in Hall thrusters have displayed the common feature of a "tail" of ions having voltage greater than that applied to the thruster discharge. Although initially dismissed as an experimental error, this tail has appeared consistently in all probe diagnostics of ion voltage. 9,10,11,12 Data obtained with the MBMS suggested that this tail may be the result of atomic collisions. A detailed discussion of the effect of atomic collisions on the measured ion voltage distributions can be found in Reference 1; a brief discussion of these effects will be presented in this paper.

For the sake of simplicity, the effect of elastic collisions on the ion voltage distribution will be discussed within the approximation of hard-sphere interactions. The differential cross section for a hard-sphere collision is $\mathrm{d}_{\mathrm{m}}{ }^{2} / 4$, where $\mathrm{d}_{\mathrm{m}}$ is the effective hardsphere diameter. Considering only binary collisions and denoting the collision scattering angle as $\theta$, the fact that the differential cross section is constant stipulates that all values of $\theta$ are equally possible in a hard-sphere collision. Therefore hard-sphere collisions produce atoms with post-collision trajectories randomly distributed over all directions.

The energy lost by a particle of "type 1" due to an elastic collision with a particle of "type 2 " is a function of the scattering angle, $\theta$ according to

Eqn. 8

$$
\Delta \mathrm{E}=\mathrm{E}_{\text {coll }} \frac{\mathrm{m}_{\mathrm{l}}}{\mathrm{m}_{2}} \sin ^{2} \frac{\theta}{2}
$$


where $\mathrm{m}_{1}$ and $\mathrm{m}_{2}$ denote the particle masses and $\mathrm{E}_{\text {coll }}$ is the energy of the collision. The interactions of relevance to this study involve particles of identical masses, such that $m_{1}=$ $\mathrm{m}_{2}$. Using $E_{1}$ and $E_{2}$ to represent the pre-collision kinetic energies, and $E_{1}{ }^{\prime}$ and $E_{2}{ }^{\prime}$ representing the post-collision energies yields

Eqn. 9

$$
E_{1}^{\prime}=E_{1}+\left(E_{2}-E_{1}\right) \sin ^{2} \frac{\theta}{2}
$$

The effects of such an interaction can be easily envisioned by analyzing the extreme cases: for glancing collisions in which $\theta=0, E_{1}{ }^{\prime}=E_{1}$ and the particles are unaffected; for head-on collisions in which $\theta=180$ degrees (complete backscattering) $E_{1}{ }^{\prime}=E_{2}$ and therefore $\mathrm{E}_{2}{ }^{\prime}=\mathrm{E}_{1}$. It follows that since the collisions will occur with equal probability for all values of $\theta$ between 0 degrees and 180 degrees the post-collision energy of particles " 1 " will be equally distributed between $\mathrm{E}_{1}$ and $\mathrm{E}_{2}$. Similarly, since the collisions are elastic particles " 2 " will be equally distributed over the same range of post-collision energies.

The effect of an elastic momentum transfer collision on the energy distribution is most easily conceived through the analysis of the most simple flow: collisions between two mono-energetic species. Figure 13 demonstrates the evolution of the energy distribution function for the interaction between such particles. The energy distribution of the entire two-species flow is shown at the far left with mono-energetic particles of species 1 and 2. Allowing a fraction of these particles to undergo elastic collisions produces the post-collision energy distributions of each species shown to the right. As evidenced by this figure the momentum transfer collision produces "tails" on the original pre-collision distributions filling the energy regime between the two species. The relative amplitude of these tails would be determined by the species density and collision cross section; in general the height of these tails will be much less than the height of the original precollision distribution. Interactions between two species having finite distributions of energy is more difficult to envision than the pedagogical case between mono-energetic species. For analysis of distributions having a finite width the reader is referred to Reference 1.

The relevance of the above discussion for this research lies in the fact that doubly ionized xenon accelerated through the same voltage as a singly charged ion will have twice the energy of the singly charged species. These high energy $\mathrm{Xe}^{2+}$ ions can transfer momentum to the $\mathrm{Xe}^{+}$and thus create the appearance of high-energy tails on the voltage distribution. Similarly, these collisions would produce low-energy tails on the $\mathrm{Xe}^{2+}$ distribution. As an example of this phenomena, Figure 14 shows the exact same physical processes as described in Figure 13, however the horizontal axis of Figure 14 is plotted in terms of ion voltage, which is energy-per-q.

Consider the overall ion voltage distribution displayed in the top portion of Figure 10. In light of the preceding discussion of collision tail formation, these data seem to display the trends associated with elastic momentum transfer collisions: a primary central distribution between monotonically decaying tails. Drawing inspiration from the similarity between the post-collision shape displayed in Figure 14 and the form of the ion voltage distributions near centerline in Figure 10 (along with other evidence detailed in Reference 1 ), assume that the measured voltage distribution represents the post-collision form of the situation introduced in Figure 14: a pre-collision voltage distribution of singly and doubly charged ions obeying the same shape, but with the doubly charged distribution having an amplitude of some fraction of the singly charged distribution. This situation seems reasonable for the Hall thruster since both ion species are formed and accelerated within the 
same region according to the same processes, albeit with the doubly charged ions produced at a reduced rate. Figure 15 shows such a supposed case for the ion voltage measured at $0.5 \mathrm{~m}$ from the SPT-100 at 5 degrees off centerline with an assumed pre-collision distribution of $\mathrm{Xe}^{+}$and $\mathrm{Xe}^{2+}$ chosen to match the central shape of the data, with the height of the $\mathrm{Xe}^{2+}$ distribution set at $15 \%$ of the $\mathrm{Xe}^{+}$height. Although there is no physical basis on which to choose a mathematical form for the pre-collision distribution, the nature of the elastic collision process is such that the post-collision distribution largely retains the precollision shape with the addition of tails attached to each side. It is therefore reasonable to assume that the 220 to $300 \mathrm{~V}$ regime of the measured distribution in Figure 10 reflects the same shape as the pre-collision distribution; thus, a gaussian curve-fit was chosen to represent the pre-collision distributions simply on the basis of providing a close mathematical approximation to the center portion of the data.

With the proposed pre-collision distributions of singly and doubly charged ions in hand the method of Reference 1 can be used to convolve the two finite-width distributions into their combined post-collision shape. Figure 16 shows the results of this convolution along with the sum of the two individual post-collision distributions compared to the MBMS data; in this figure the amplitude of the post-collision product distribution (height of the tail) was chosen arbitrarily to match the tail in the data; physically, this tail height would be a function of the collision cross section.

Even without the value of the elastic cross section between $\mathrm{Xe}^{+}$and $\mathrm{Xe}^{2+}$ the trends and qualitative behavior in the post-collision convolution of the assumed distribution very closely match the data curve. Specifically, the high-energy tail in the data decays in nearly the same manner as the tail in the proposed model, falling to a value of zero at the same value of voltage; the shape of the low-energy tails also reach zero at the same voltage. Thus, although the model contains no method by which to set the tail height, the overall width and trends in the proposed convolution closely follow the data.

By utilizing the species-dependent data obtained from the MBMS it is possible to evaluate the proposed elastic collision concepts discussed above more extensively. Using the TOF mode of the MBMS the species-dependent voltage distributions were measured at $0.5 \mathrm{~m}, 5$ degrees for $\mathrm{Xe}^{+}, \mathrm{Xe}^{2+}$, and $\mathrm{Xe}^{3+}$; these data were presented previously as Figure 11. Figure 17 shows a comparison between the $\mathrm{Xe}^{+}$and $\mathrm{Xe}^{2+}$ post-collision distributions derived by convoluting the assumed pre-collision gaussians (as plotted in Figure 15) compared with the MBMS-measured voltage distributions of these same species.

The similarity between the shapes computed based on the assumed pre-collision distributions convoluted according to elastic collisions and the data is striking: the $\mathrm{Xe}^{+}$ distribution data exhibits a high-energy tail that extends and decays to zero nearly identically with the computed curve, while the $\mathrm{Xe}^{2+}$ distribution displays the corresponding low-energy tail attributed to elastic collisions with $\mathrm{Xe}^{+}$that also follows the computed shape. It follows, then, that the ion voltage distribution data at 5 degrees off centerline, 0.5 $\mathrm{m}$ from the thruster is consistent with the description of the phenomena attributed to elastic momentum transfer collisions between singly and doubly charged plume beam ions having a gaussian-like pre-collision distribution: the high-voltage tail is formed by singly charged xenon ions that have gained momentum from the higher-energy doubly charged ions, while the low-energy tail is the corresponding appearance of doubly-charged ions which have lost energy through elastic collisions. This explanation seems plausible since the assumed precollision distributions shown in Figure 15 resemble the intuitive shape of the ion voltage distribution that would be expected to form within the plasma discharge described by the trends shown in Figure 12: ion voltages ranging from 200 to $300 \mathrm{~V}$ with no ions exceeding the applied discharge voltage. Although the pre-collision distributions of Figure 15 exhibit a small population of ions greater than $300 \mathrm{~V}$, it must be remembered that these 
shapes were simply assumed with no physical justification other than agreement with data. The curve-fit representing the pre-collision shape complied with the data only up to about $290 \mathrm{~V}$ : the portion of the pre-collision distribution greater than $300 \mathrm{~V}$ is therefore intangible.

The comparisons of Figure 17 show exceptional agreement for the singly ionized xenon distributions, whereas the doubly charged distributions display a slight offset. The reason for this disagreement is likely to arise from the acceleration scenario discussed previously: rather than having the same pre-collision shape and location as the $\mathrm{Xe}^{+}$ distribution as assumed, the doubly-charged ions are likely to be formed further downstream in the thruster acceleration layer and would thus have a pre-collision voltage distribution that was shifted towards lower voltages. This accounts for the offset apparent in Figure 17. However, the breadth of the low-energy tail on the $\mathrm{Xe}^{2+}$ distribution is determined by the pre-collision voltage distribution of $\mathrm{Xe}^{+}$and therefore shows good agreement with the data in spite of the main peak offset.

It is noteworthy that the ion voltage distribution for $\mathrm{Xe}^{3+}$ as displayed in Figure 11 displayed no tail of ions with voltages greater than that applied to the plasma discharge. This fact is supported by the proposed collisional process responsible for the high-energy tail on the $\mathrm{Xe}^{+}$distribution: since no significant evidence for the existence of $\mathrm{Xe}^{4+}$ was uncovered in the time-of-flight spectra there should be no ion with greater energy than $\mathrm{Xe}^{3+}$. Thus there would exist no process by which a triply ionized xenon atom could gain more energy than that applied to the thruster discharge.

\section{Conclusions}

The MBMS provided a direct measurement of the ionization fraction of the propellant within the SPT-100 plume independent of a model describing the plasma equilibrium state. These values compared very well with the same quantities measured previously using an emission spectroscopic techinque. Thus, it can be confidently assumed that the plume plasma in the SPT-100 is composed of $89 \% \mathrm{Xe}^{+}, 11 \% \mathrm{Xe}^{2+}$, and less than $1 \% \mathrm{Xe}^{3+}$.

In addition to an analysis of the majority propellant ions, the MBMS confirmed the existence of entrained background gasses caused by vacuum facility imperfections. These gasses were evidenced by the appearance of nitrogen, oxygen, and water vapor within the high energy plasma mass species. Ground-test facility interactions were also manifested by the appearance of carbon ions within the plume caused by sputtering of protective graphite used in proximity of the thruster.

The near-centerline region of the plume was seen to exhibit considerable evidence of elastic momentum transfer collisions between singly and doubly charged propellant ions. Although these collisions could have occurred anywhere along the path length from the discharge chamber to the MBMS detector, it is most likely that they originated from the region in which the reactant density is greatest, i.e. near the thruster discharge chamber. Furthermore, the behavior of the post-collision voltage distributions stipulates that the elastic collisions between $\mathrm{Xe}^{+}$and $\mathrm{Xe}^{2+}$ must have occurred between high-voltage ions, i.e. these collisions must have occurred downstream of the acceleration zone in order to produce the tail lengths displayed in the data. It follows, then, that the highest probability of elastic collisions between beam ions producing the structure seen in the data will occur immediately downstream of the thruster acceleration zone, or near the thruster exit plane. 
These collisions are not due to a facility interaction effect, rather they are a manifestation of the basic thruster operation.

\section{Acknowledgments}

This research benefited from the generous support of the Air Force Office of Scientific Research (AFOSR) represented by Dr. Mitat Birkan, the NASA-Lewis Research Center with equipment grants administered by Mr. John Sankovic, and support from the NASA-Johnson Space Center under the direction of Mr. Richard Barton. The unique opportunity to evaluate a state-of-the-art thruster was made available by a generous equipment loan from Mr. Mike Day of the Space Systems/Loral company. This support is gratefully acknowledged. Additionally, the authors would like to thank technicians Warren Eaton, Terry Larrow, Gary Gould, and Dave McLean for assitance with hardware fabrication. The first author would also like to thank the research staff of PEPL, namely, Colleen Marrese, Frank Gulczinski, James Haas, Sang-wook Kim, and George Williams for their discussions in the preparation of this manuscript. 


\section{References}

1 King, L., Transport Properties and Mass Spectral Measurements in the Plasma Exhaust Plume of a Hall-effect Space Propulsion System, Doctoral Dissertation, University of Michigan Dept. of Aerospace Engineering, Published through University Microfilm Internat'l, 1998.

2 Manzella, D., "Stationary Plasma Thruster Plume Emissions," IEPC-93-097, 23rd International Electric Propulsion Conference, Seattle, WA, Sept. 13-16, 1993.

Manzella, D., "Stationary Plasma Thruster Ion Velocity Distribution," AIAA-943141, 30th AIAA/ASME/SAE/ASEE Joint Propulsion Conference, Indianapolis, IN, June 27-29, 1994.

$4 \quad$ King, L.B., and Gallimore, A.D., "Ion Energy Diagnostics in the Plume of an SPT-100 from Thrust Axis to Backflow Region," AIAA-98-3641, 34th AIAA/ASME/SAE/ASEE Joint Propulsion Conference, Cleveland, OH, July 13$15,1998$.

$5 \quad$ Pollard, J., "Plume Angular, Energy, and Mass Spectral Measurements with the T5 Ion Engine," AIAA-95-2920, 31st AIAA/ASME/SAE/ASEE Joint Propulsion Conference, San Diego, CA, July 10-12, 1995.

Lide, D.R., Ed., Handbook of Chemistry and Physics, 75th Edition, CRC Press, Ann Arbor, 1994.

7 Bishaev, A., and Kim, V., "Local Plasma Properties in a Hall-current Accelerator with an Extended Acceleration Zone," Soviet Physics-Technical Physics, Vol. 23, Sept. 1978, pp. 1055-1057.

8 Baranov, V., Nazarenko, Y., Petrosov, V., Vasin, A., and Yashnov, Y., "Energy Model and Mechanisms of Acceleration Layer Formation for Hall Thrusters," AIAA-97-3047, 33rd AIAA/ASME/SAE/ASEE Joint Propulsion Conference, Seattle, WA, July 6-9, 1997.

Bugrova, A., Desyatskov, A., and Kharchevnikov, V., "Experimental Determination of Ion Energy at the Outlet of SPT-ATON," IEPC-95-47, 24th International Electric Propulsion Conference, Moscow, Russia, 1995.

10 King, L.B., Gallimore, A.D., and Marrese, C.M., "Transport-property Measurements in the Plume of an SPT-100 Hall-effect Thruster," AIAA Journal of Propulsion and Power, Vol. 14, No. 3, May-June, 1998, pp. 327-335.

11 Myers, R., and Manzella, D., "Stationary Plasma Thruster Plume Characteristics," IEPC-93-096, 23rd International Electric Propulsion Conference, Seattle, WA, Sept. 13-16, 1993.

Absalamov, S., Andreev, V., Colbert, T., Day, M., Egorov, V., Gnizdor, R., Kaufman, H., Kim, V., Korakin, A., Kozubsky, K., Kudravzev, S., Lebedev, U., Popov, G., and Zhurin, V., "Measurement of Plasma Parameters in the Stationary Plasma. Thruster (SPT-100) Plume and its Effect on Spacecraft 
Components," AIAA-92-3156, 28th AIAA/SAE/ASME/ASEE Joint Propulsion Conference, Nashville, TN, July 6-8, 1992. 


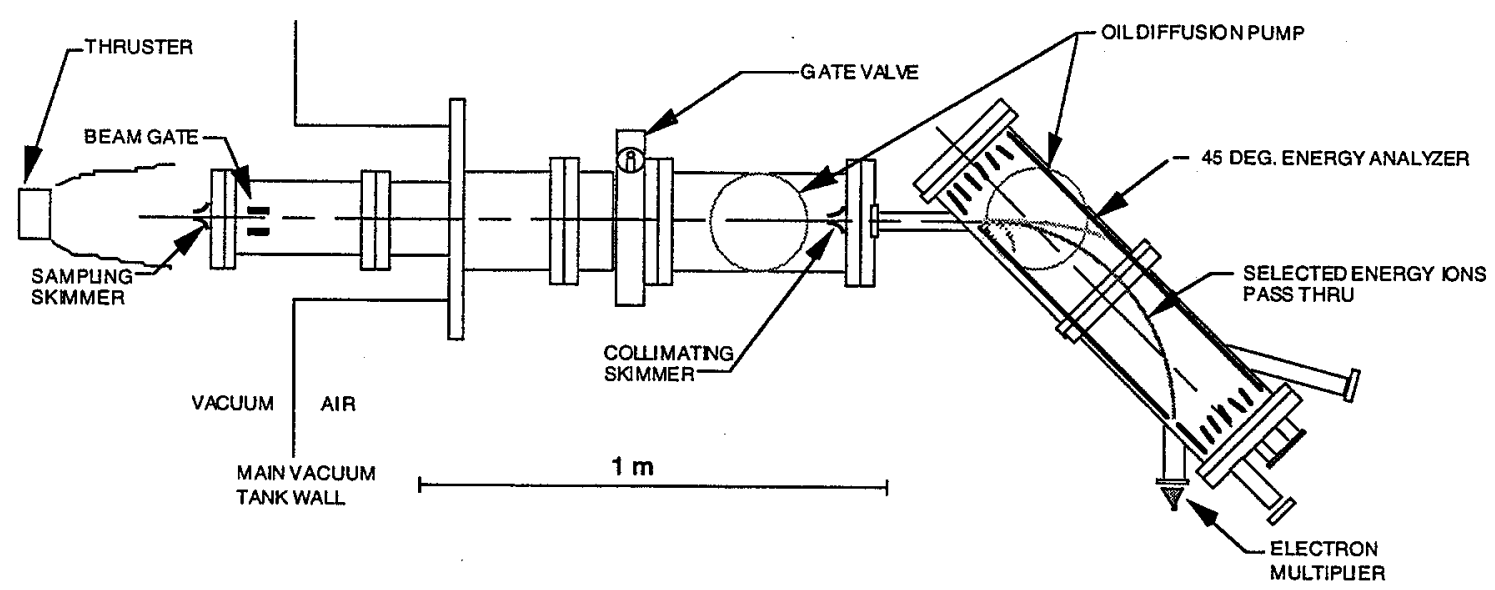

Figure 1. Schematic of overall configuration of MBMS apparatus showing orientation to main vacuum chamber, thruster mount, and scale size.

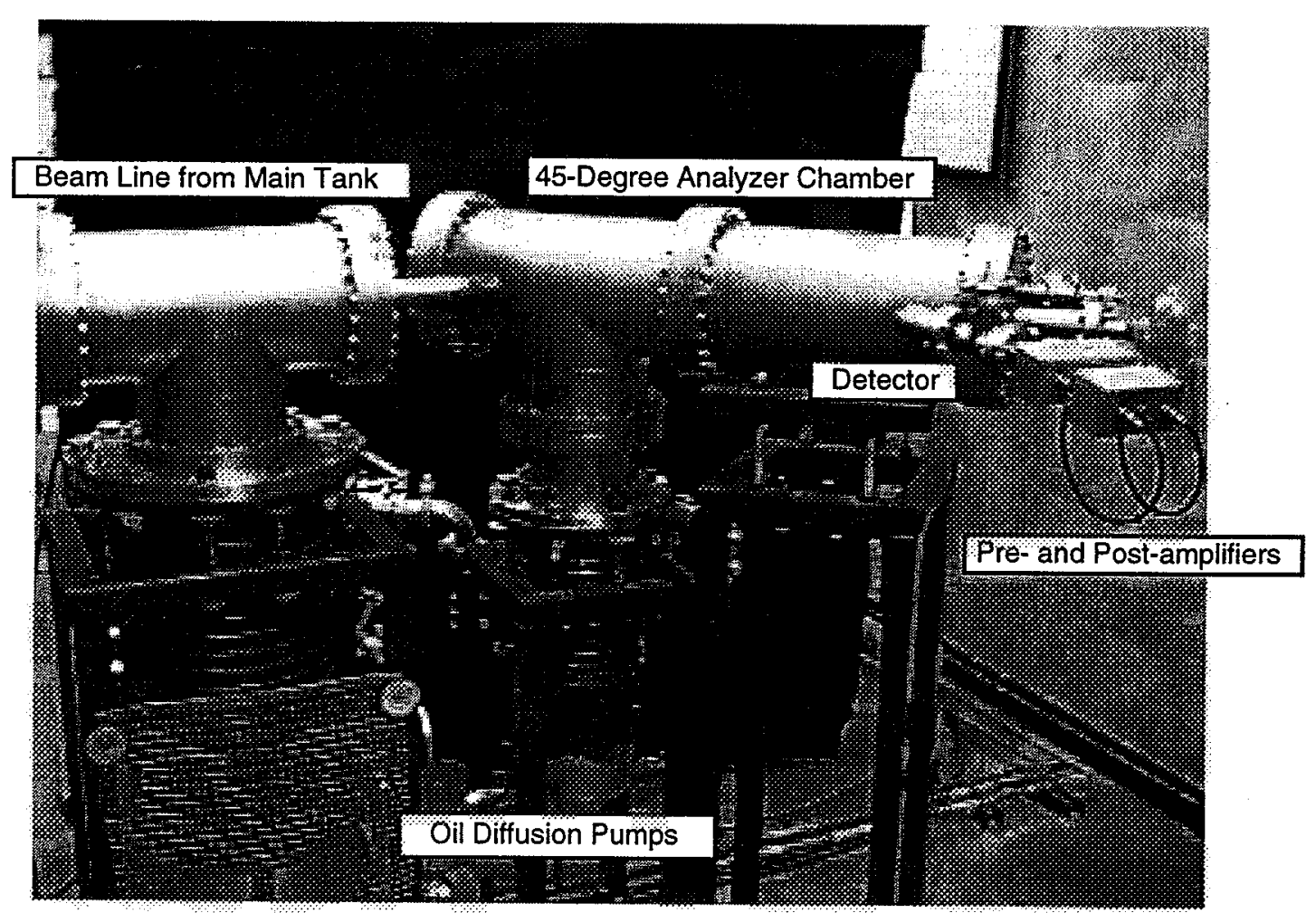

Figure 2. Photograph of MBMS instrument showing electrostatic analyzer chamber and electron multiplier detector port. 


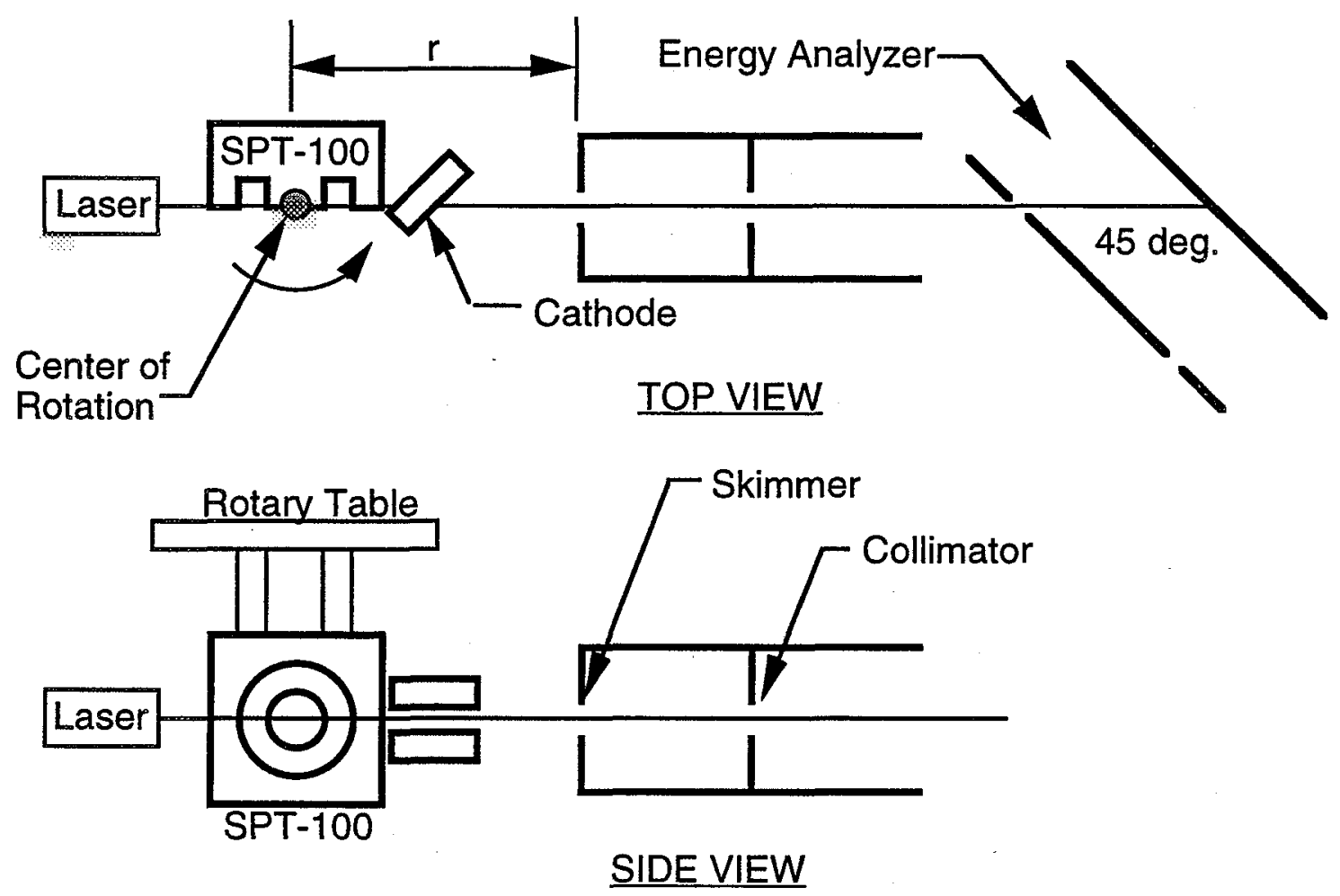

Figure 3. Experimental set-up diagram showing rotary thruster mount and laser alignment of beam line. 


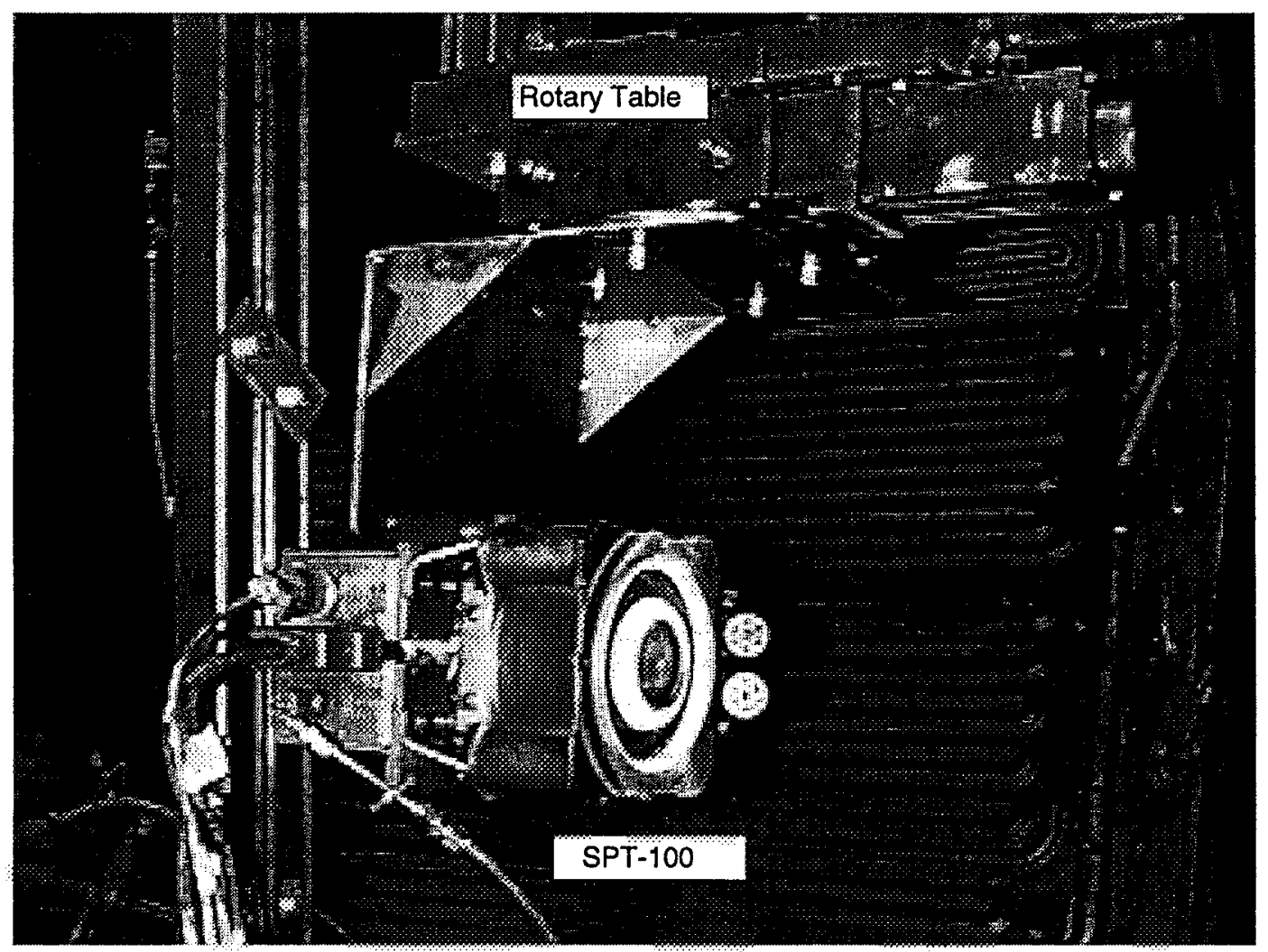

Figure 4. Photograph showing SPT-100 mount to rotary table for MBMS characterization. 


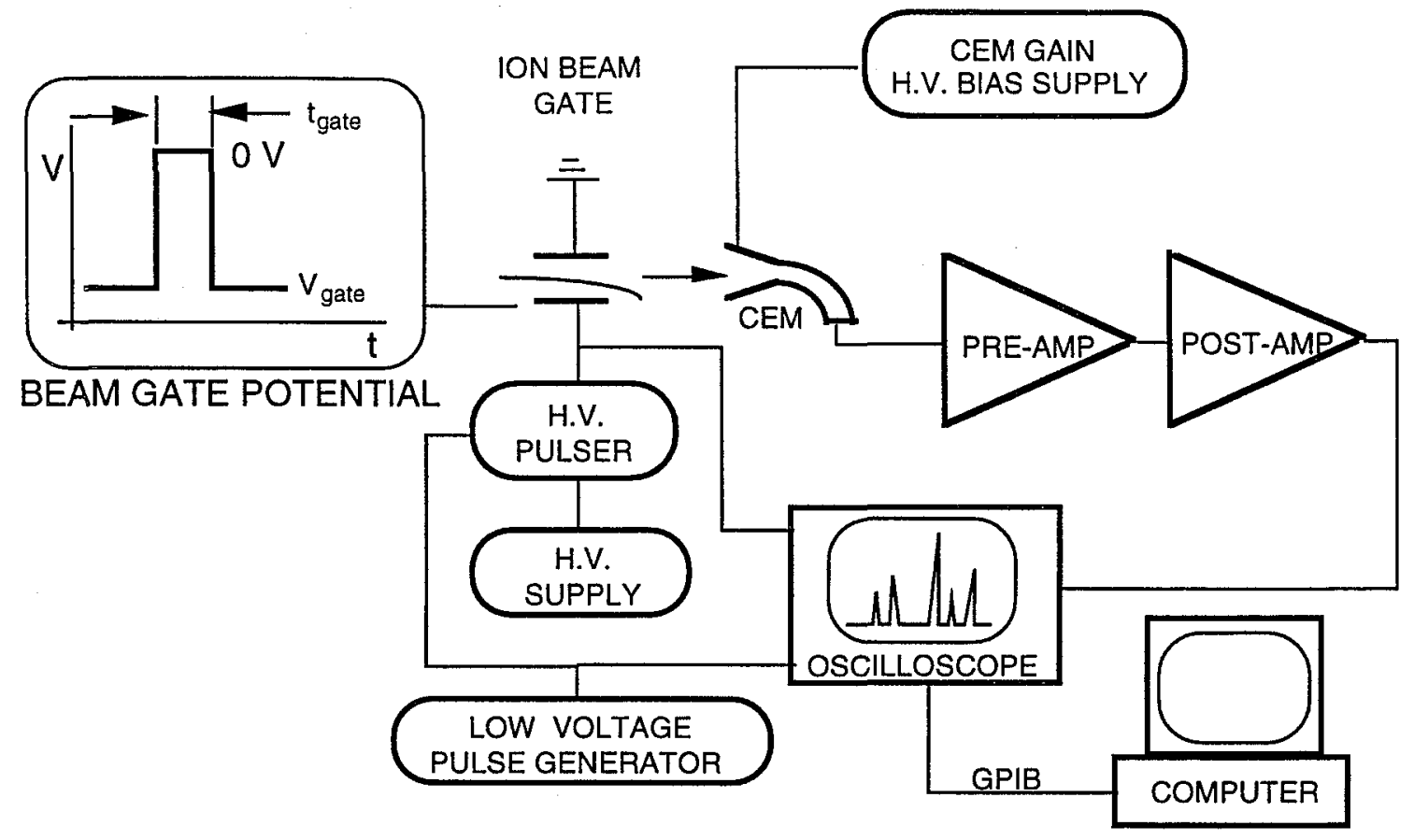

Figure 5. Electrical schematic of controlling electronics and data system for TOF spectrometer.

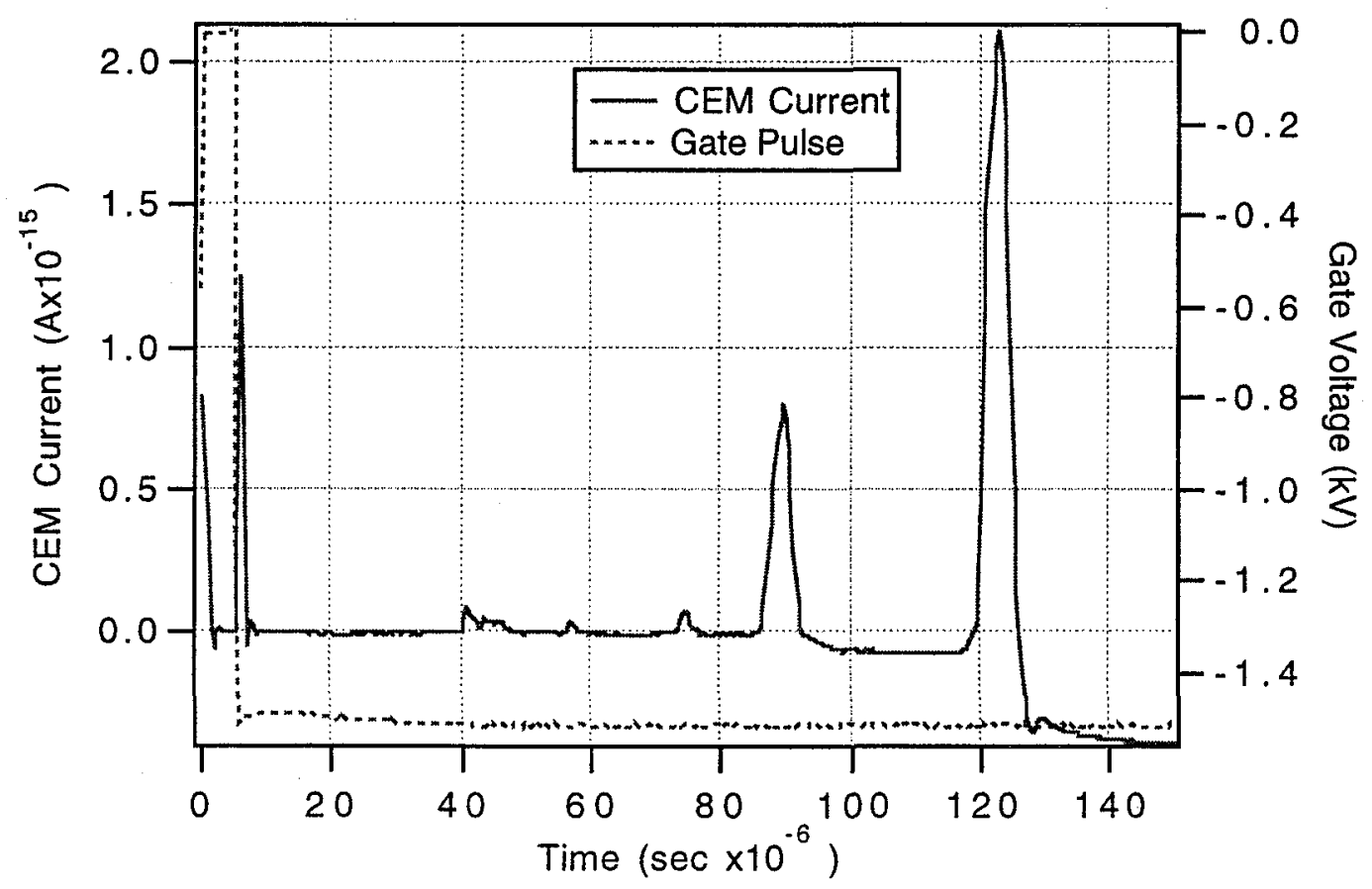

Figure 6. Typical TOF spectra for $280 \mathrm{~V}$ ions at $0.5 \mathrm{~m}$ radius from the $S P T-100$ for the point 5 degrees off thruster axis. The dashed line represents the beam gate potential and corresponds to the right axis. The solid line reflects the CEM output current, which is scaled on the left axis. 


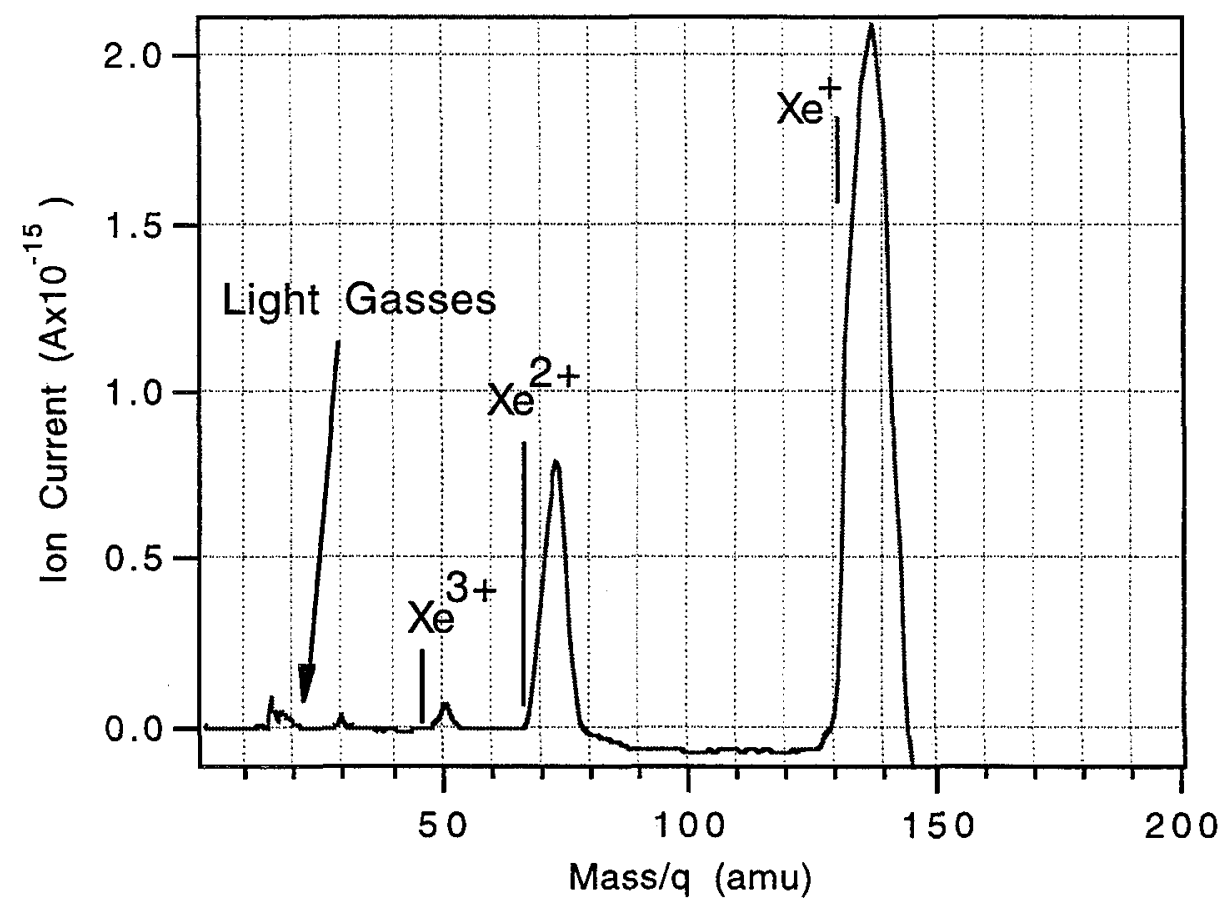

Figure 7. Typical mass spectra for $260 \mathrm{~V}$ ions at $0.5 \mathrm{~m}$ radius 5 degrees off thrust axis. 


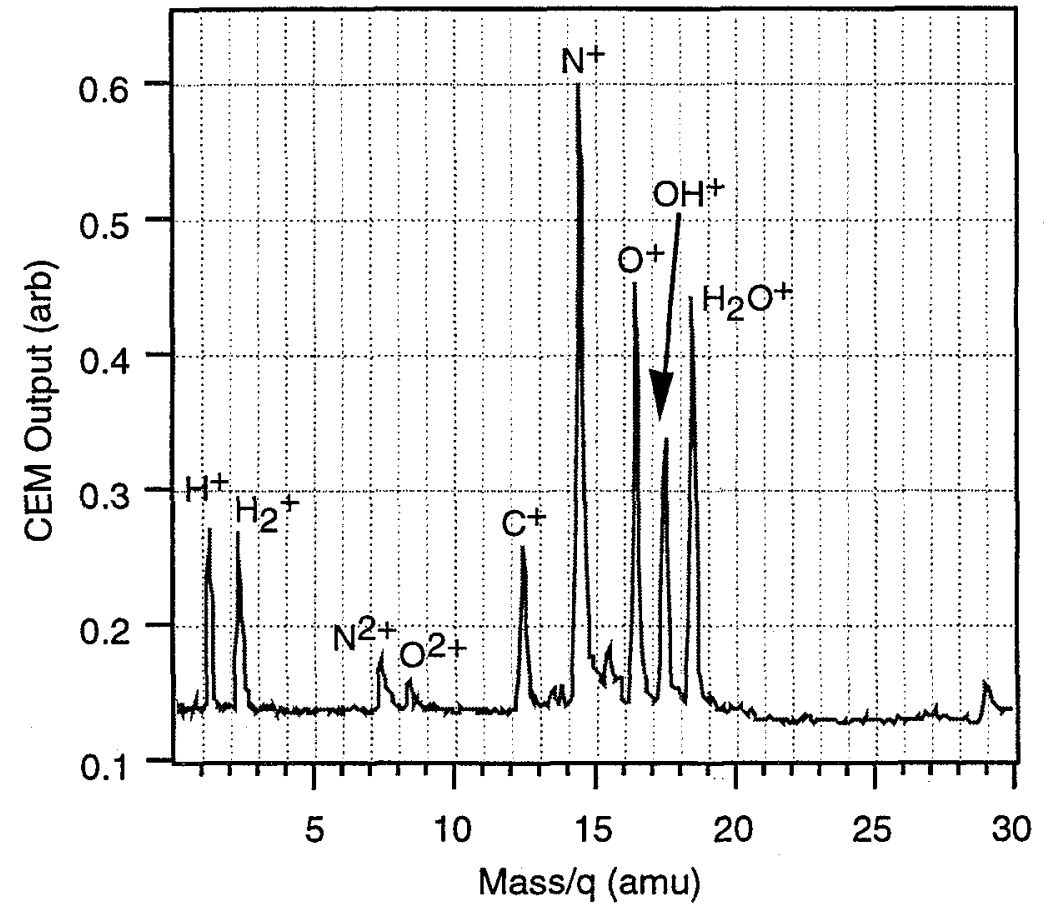

Figure 8. Minor species identification for $260 \mathrm{~V}$ ions at $0.5 \mathrm{~m}$ from the SPT-100 for the point 5 degrees off thrust axis. 


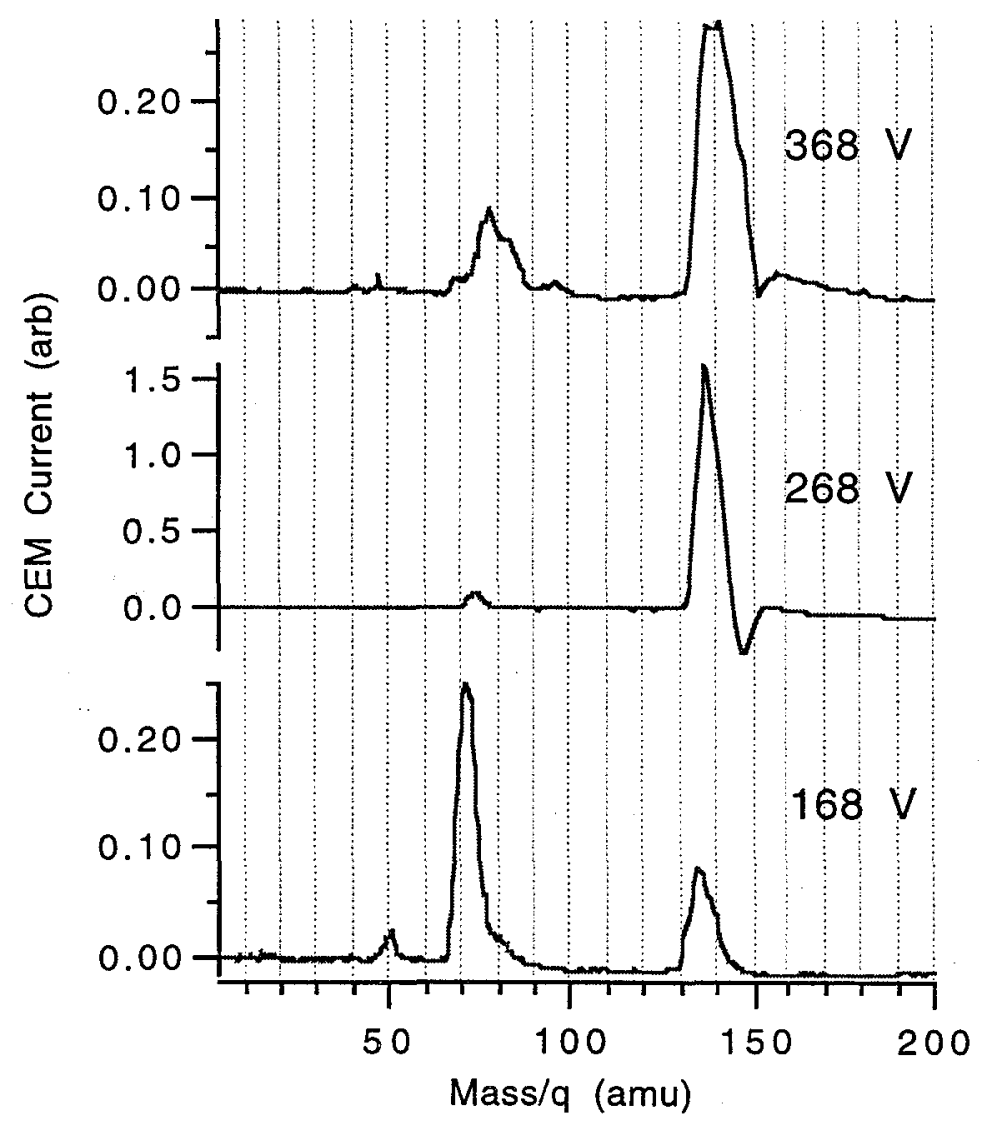

Figure 9. Mass spectra for evaluation of xenon ionization states for ions with 180,280 , and $380 \mathrm{~V}$ acceleration in the SPT-100 at $0.5 \mathrm{~m}$ radius, 5 degrees off thrust axis. 


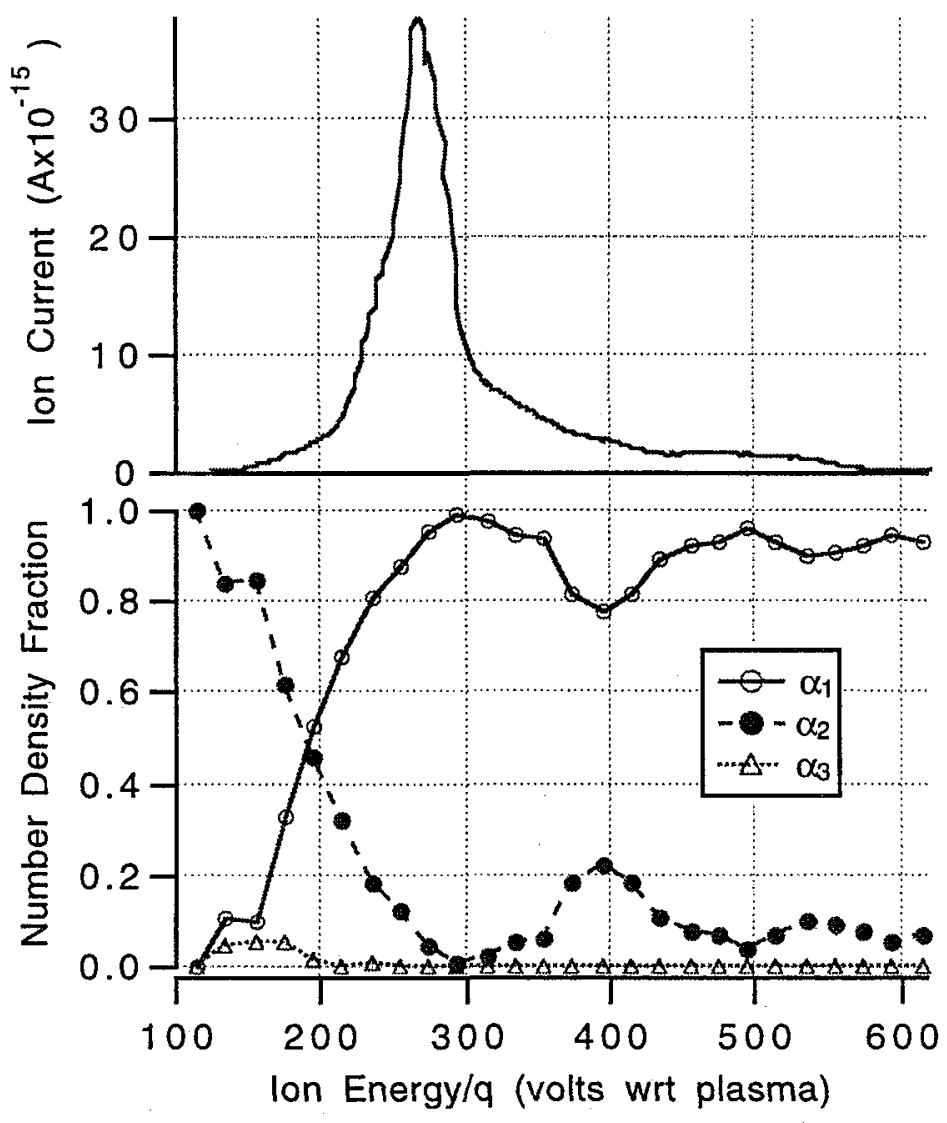

Figure 10. Number density fractions of $\mathrm{Xe}^{+}, \mathrm{Xe}^{2+}$, and $\mathrm{Xe}^{3+}$ as a function of ion energy at $0.5 \mathrm{~m}$ radius and 5 degrees off thrust axis in the SPT-100. Also shown in the top curve is the value of total ion current as a function of ion voltage for comparison of total density. 


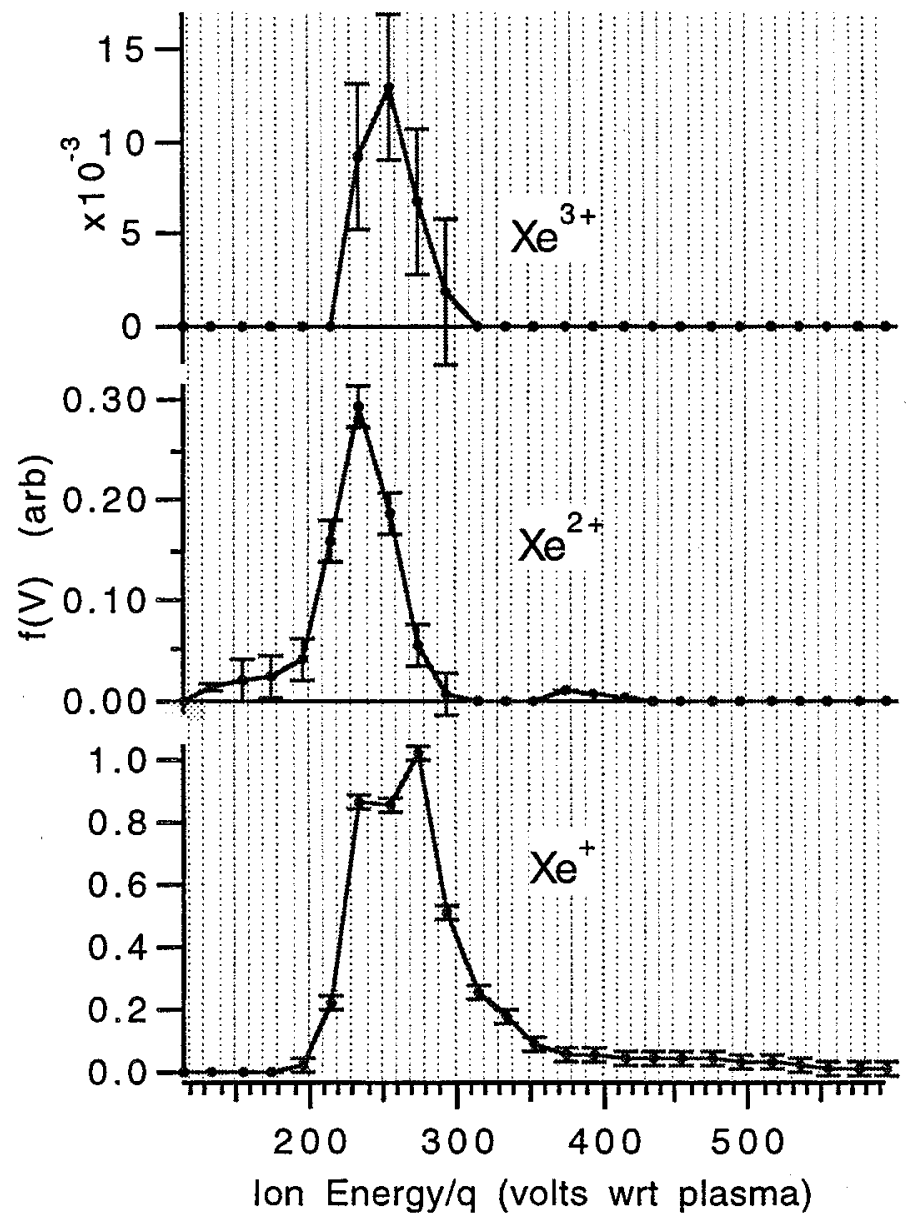

Figure 11. The ion voltage distribution function for each propellant ionization species in the SPT-100 at $0.5 \mathrm{~m}$ radius and 5 degrees off thrust axis. 


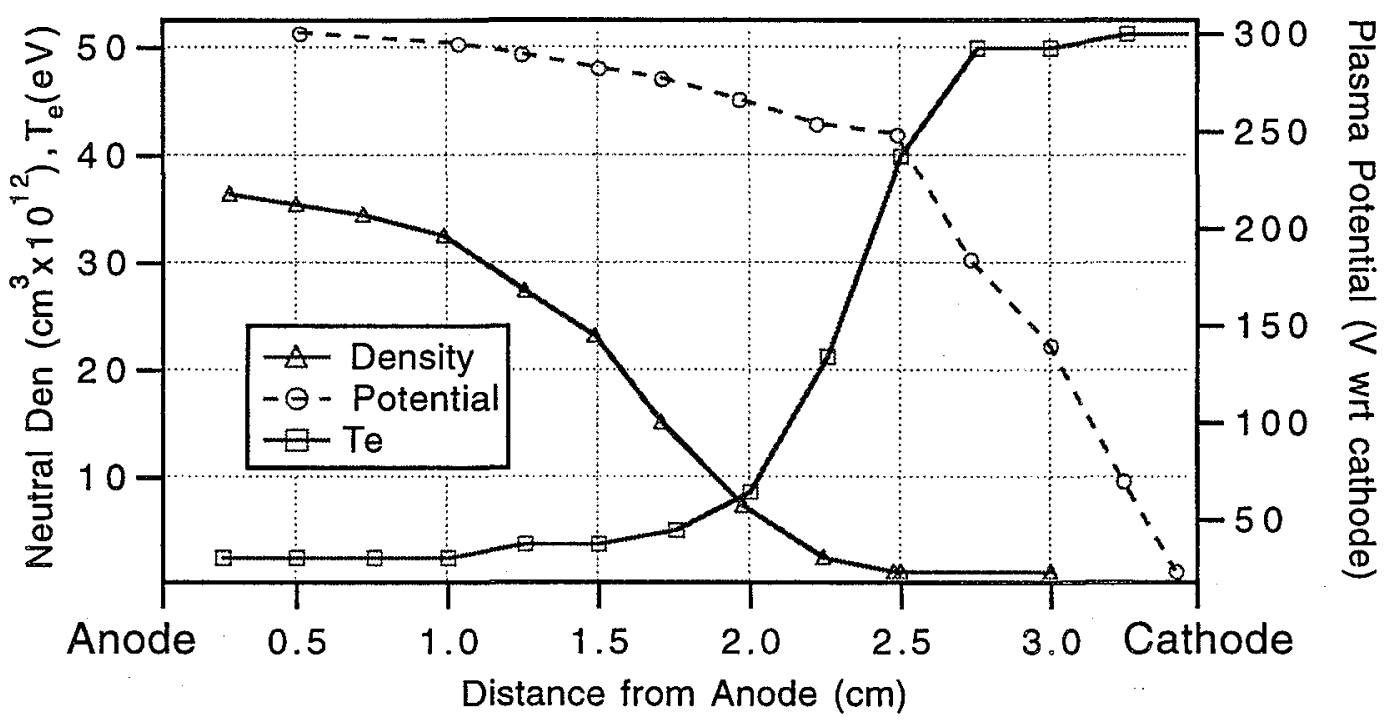

Figure 12. Results of Baranov model ${ }^{8}$ of plasma parameters in Hall thruster discharge chamber including neutral atom density (left axis), electron temperature (left axis), and plasma potential (right axis). 


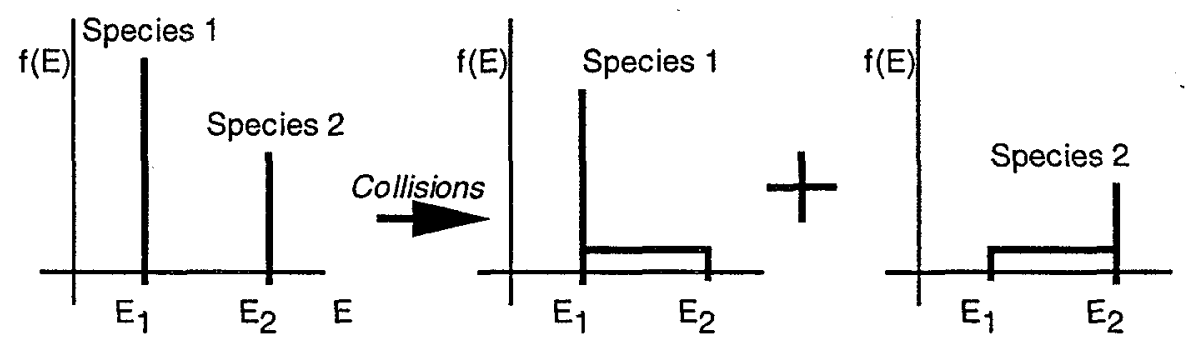

Figure 13. Illustration of tail formation mechanism in the energy distribution through elastic collisions between two mono-energetic species.

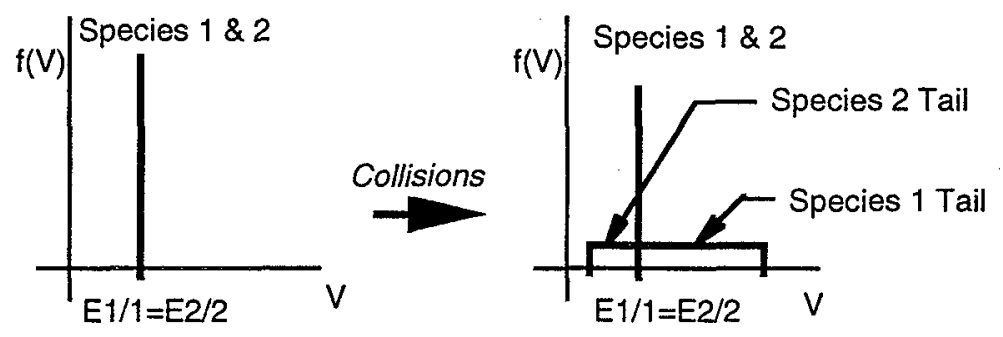

Figure 14. Voltage equivalent distribution of the tail-producing collision scenario depicted in Figure 13. Species 1 represents singly ionized atoms while Species 2 represents doubly charged ions accelerated through the same voltage. The tails are produced through momentum transfer collisions. 


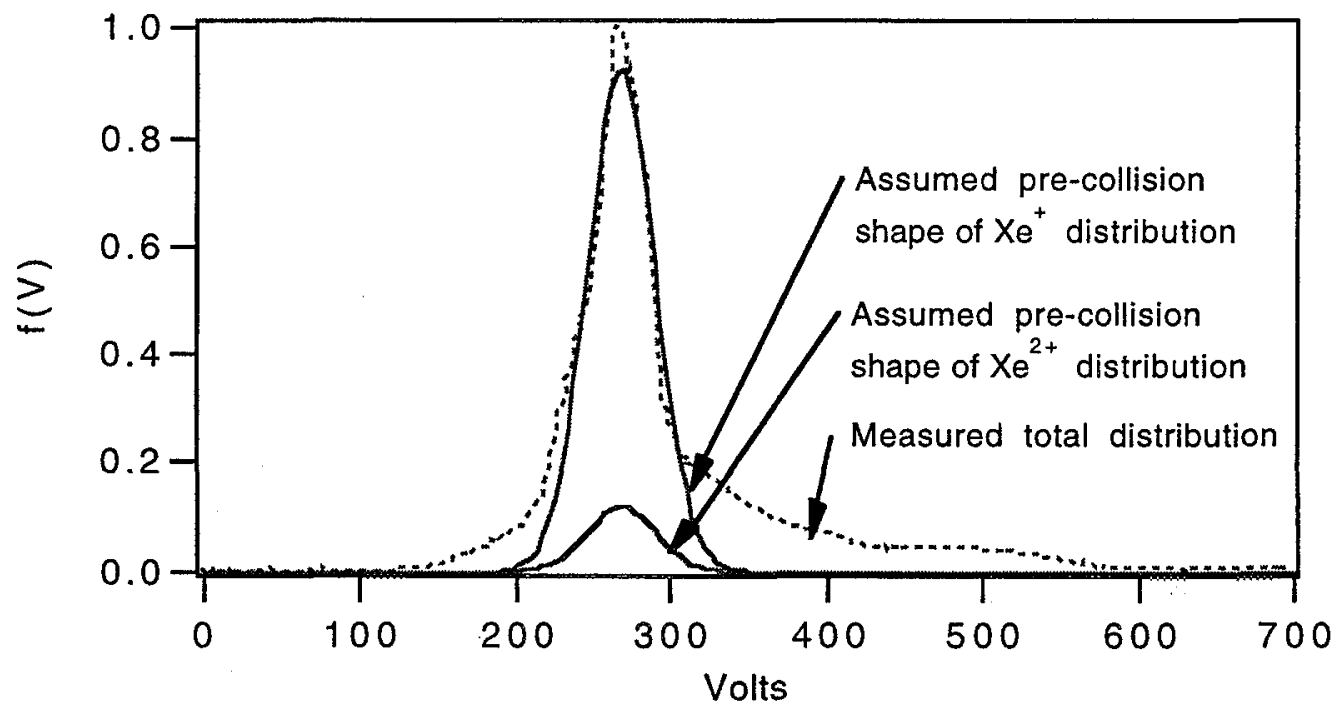

Figure 15. Total ion voltage distribution measured at $0.5 \mathrm{~m}$ from the SPT- 100 at 5 degrees off thruster centerline with proposed forms of the pre-collision distributions of singly and doubly charged ions. 


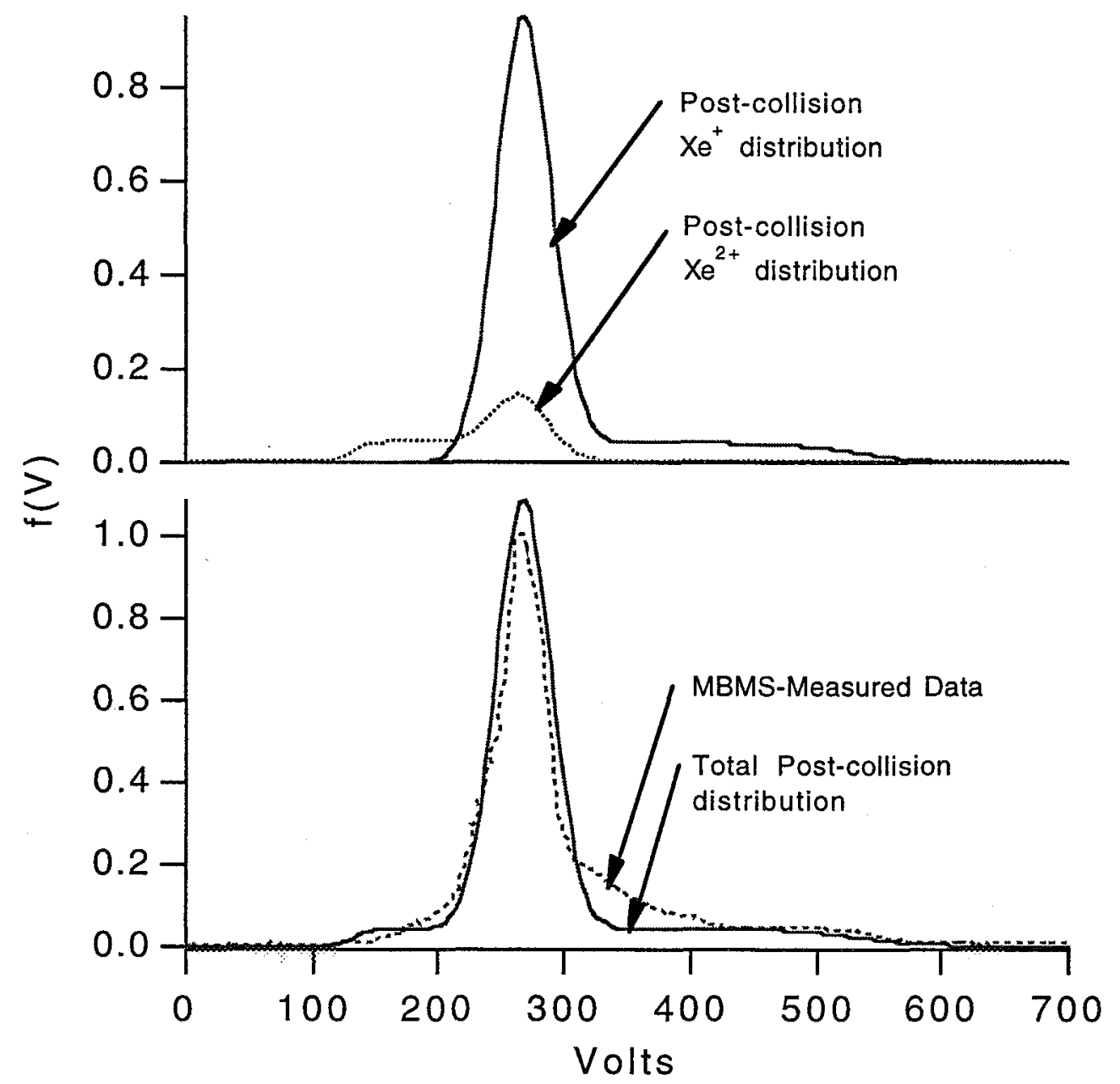

Figure 16. Convolution of assumed pre-collision gaussian distributions of $\mathrm{Xe}^{+}$and $\mathrm{Xe}^{2+}$ into their combined post-collision shape compared with MBMS-measured data at $0.5 \mathrm{~m}$ radius from the SPT-100 and 5 degrees off centerline. 


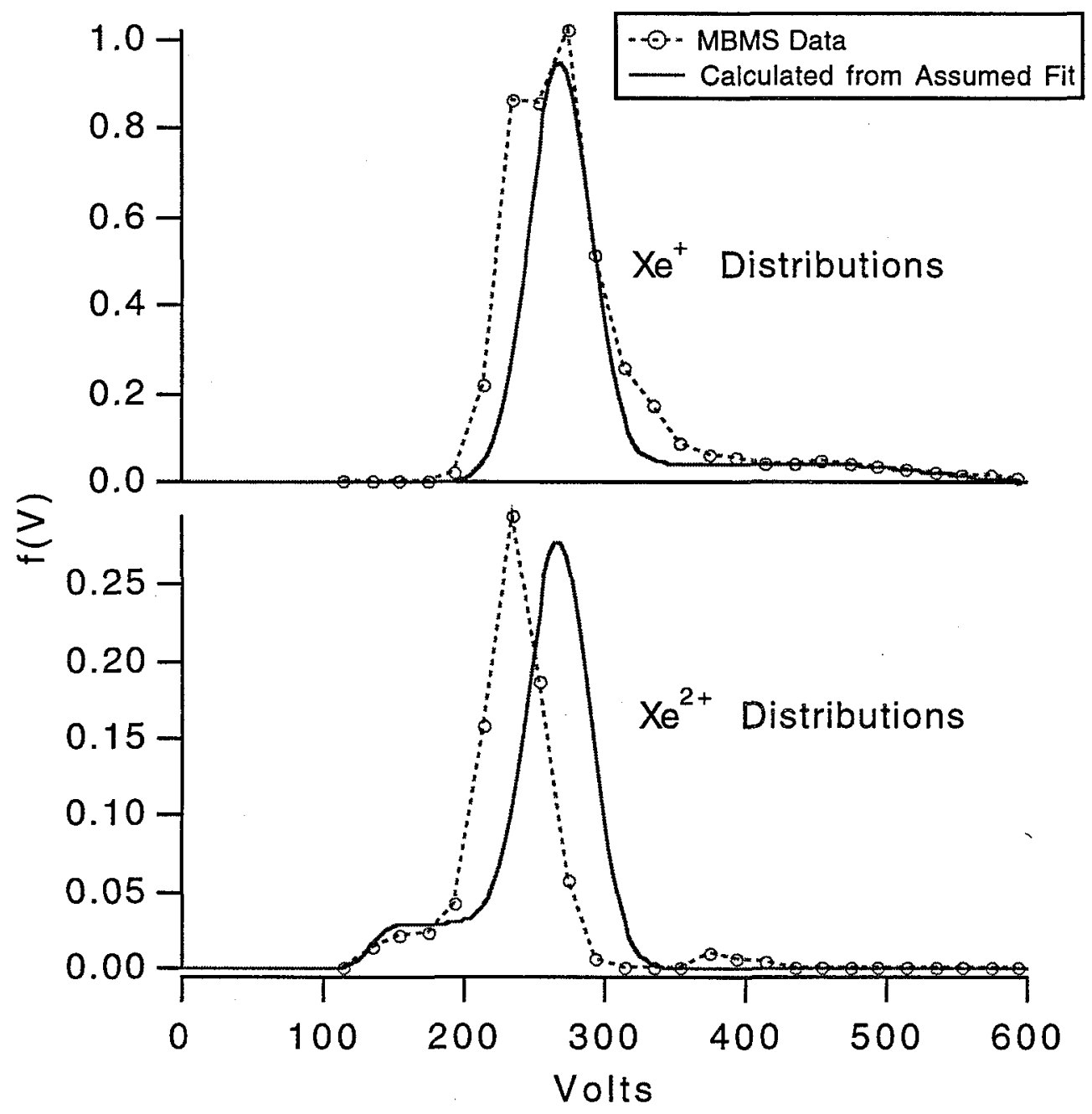

Figure 17. Comparison of MBMS data with convolution of proposed pre-collision gaussians for $\mathrm{Xe}^{+}$and $\mathrm{Xe}^{2+}$ at $0.5 \mathrm{~m}$ from the SPT-100, 5 degrees off centerline. 